\title{
Burnout, docencia e investigación en profesores universitarios de la Facultad de Ciencias Empresariales de Perú y España
}

\author{
Burnout, teaching and research in university professors of the Faculty of Business \\ Sciences in Peru and Spain
}

\section{Rosario Margarita Yslado Méndez (rysladom@unasam.edu.pe)}

http://orcid.org/0000-0001-6820-8607

Edwin Hernán Ramírez Asís (ehramireza@unasam.edu.pe)

https://orcid.org/0000-0002-9918-7607

\author{
Marcos Rosamel Espinoza Maguiña (mespinozam@unasam.edu.pe) \\ https://orcid.org/0000-0002-2570-9273 \\ Universidad Nacional Santiago Antúnez de Mayolo (Perú)
}

http://dx.doi.org/10.12795/EDUCADE.2020.i1 1.02

\begin{abstract}
RESUMEN: Diferentes estudios sobre el síndrome de burnout (SB) en profesores universitarios reportan relaciones entre esta psicopatología laboral y las variables sociodemográficas, personales, laborales y extralaborales; sin embargo, son escasos los estudios transculturales sobre el SB por actividades de docencia e investigación. El objetivo del estudio fue analizar las diferencias del síndrome de burnout, sus dimensiones y según funciones sustantivas de docencia e investigación, en los profesores de las Facultades de Ciencias Empresariales (Economía, Contabilidad, Administración y Turismo) de la Universidad de Sevilla (US) de España y la Universidad Nacional Santiago Antúnez de Mayolo (UNASAM) de Perú. Es una investigación de enfoque cuantitativo, de nivel básico y descriptivo, de diseño no experimental, transversal y descriptiva comparativa; se utilizó el cuestionario adaptado del Maslach Burnout Inventory (MBI), que mide el SB asociado a docencia e investigación. La muestra estuvo compuesta por 68 profesores de las universidades públicas investigadas. Los resultados indican que hay diferencias significativas según la universidad para el SB en sus dimensiones de cansancio emocional por docencia, despersonalización por docencia e investigación y en baja realización personal por docencia e investigación. En el caso de la diferencia según el sexo, se identificó que existe diferencia significativa en la baja realización personal por investigación en los docentes de la Universidad de Sevilla. En lo que respecta a las diferencias según la edad, no se encontró diferencias significativas. En conclusión, los profesores de la universidad peruana presentan niveles significativamente más altos de SB por docencia e investigación, en comparación con los profesores de la universidad española.
\end{abstract}

PALABRAS CLAVE: Burnout, docencia, investigación, profesores universitarios.

\begin{abstract}
Different studies on burnout syndrome (SB) in university professors report relationships between this occupational psychopathology and socio-demographic, personal, occupational and extra-occupational variables; however, cross-cultural studies on SB by teaching and research activities are scarce. The aim of the study was to analyze the differences in burnout syndrome according to its dimensions, by teaching and research activity, among professors of the Faculties of Business Sciences (Economics, Accounting, Administration and Tourism) of the University of Seville (US) of Spain and Santiago Antúnez de Mayolo National University (UNASAM) of Peru, according to the university of origin and the sociodemographic variables of sex and age. It is a research of quantitative approach, of basic and descriptive level, of non-experimental,
\end{abstract}


transversal and comparative descriptive design; the questionnaire adapted from the Maslach Burnout Inventory (MBI) by Arquero and Donoso (2006) was used, which measures the SB associated to teaching and research. The sample was composed of 68 professors from the public universities researched. The results indicate that there are significant differences according to the university for SB in its dimensions of emotional fatigue for teaching, depersonalization for teaching and research and in low personal fulfillment for teaching and research. In the case of the difference according to sex, it was identified that there is a significant difference in the low personal realization by research in the teachers of the University of Seville. With regard to the differences according to age, no significant differences were found. In conclusion, the professors of the Peruvian university present significantly higher levels of SB by teaching and research, compared to the professors of the Spanish university.

KEYWORDS: Burnout, teaching, research, university professors.

Artículo. Recibido: 10-05-20 - Aceptado: 20-12-20

Licencia Creative Commons BY NC ND $\cdot 2020 \cdot$ Universidad de Sevilla - AECA

\section{INTRODUCCIÓN}

En el presente siglo los cambios profundos, acelerados y constantes suscitados a nivel mundial, de tipo económico, político, social, cultural y tecnológico generan presiones en las organizaciones laborales, como las universidades; por lo tanto, el profesor universitario de cualquier país, en mayor o menor grado está expuesto a riesgos psicosociales que afectan su salud ocupacional, tales riesgos son: excesivas exigencias laborales, sobrecarga laboral, espacios inadecuados, bajo apoyo social, inestabilidad laboral, disminución de recursos, aumento de la demanda, insuficiente tiempo y bajas remuneraciones que compense los esfuerzos-presiones y carga de trabajo (docencia, investigación, publicación de calidad y otros), que configuran un contexto laboral propicio para la emergencia del estrés laboral y el síndrome de burnout (Avargues y Borda, 2010; Botero, 2012; Fajardo, Montejo, Molano, Hernández y Quintero, 2013; García, Iglesias, Saleta y Romay, 2016; Gómez, Perilla y Hermosa, 2019).

El ejercicio de la docencia universitaria en Iberoamérica, de una parte, puede ser fuente de desarrollo y realización personal; pero de otra puede generar diferentes grados de tensión en los profesores de las Facultades de Ciencias Empresariales, que van desde el estrés laboral hasta el burnout. Las consecuencias del burnout en los profesores, se evidencian a través del decremento de la salud mental y ocupacional, disminución del adecuado desempeño docente, deterioro de la calidad de la enseñanza e investigación con pertinencia para su entorno, aumento de la intención de abandono de la profesión, insatisfacción laboral, estancamiento de metas y expectativas personales y laborales; problemas que se agudizan, si se asocian a menor calidad de vida en el contexto laboral y familiar (Arquero y Donoso, 2013; Conceição, Pastore da Silveira, Barbosa de Sá, De Barros, Silva y De Barros, 2015; Díaz, 2014; Mena, 2010; Moreno-Jiménez, Garrosa y Rodríguez, 2009; Rivera, Segarra y Giler, 2018).

Las nuevas políticas públicas para la educación superior son implementadas en las universidades del Perú y España, en un contexto laboral de cambios vertiginosos, cuyas condiciones laborales, recursos y salarios no han mejorado significativamente para responder de manera adecuada al reto de asegurar la calidad académica, que es evaluada y controlada por el Estado (Monarca y Prieto, 2018; Ocaña, Rodríguez, 
Urbina y Sánchez, 2015). Los profesores universitarios están expuestos a mayor tensión y sobrecarga laboral en el trabajo, debido a la fragmentación de sus actividades y diversas responsabilidades, tales como: realizar innovaciones pedagógicas; incorporar recursos informáticos; implementar metodologías alternativas de enseñanza y aprendizaje; adoptar cambios curriculares; continua actualización y perfeccionamiento; aceptar la primacía de la investigación y su incentivación; enfrentar problemas emergentes sociales, organizacionales y con los estudiantes, responsabilidades y funciones para las cuales no fue preparado en su formación tradicional (Villamar, Juárez, Gonzáles y Osnaya, 2019).

Los aspectos mencionados en el párrafo precedente inciden en la etiopatogenia del SB de los profesores, además de otros aspectos: primero, los factores individuales como el tipo de personalidad, resiliencia, locus de control, inteligencia emocional, autoeficacia, salud mental y otros). Segundo, los factores intralaborales, como alumnos exigentes y conflictivos, individualismo de colegas, desilusión gradual respecto al trabajo, clima laboral desfavorable, percepción de inequidad e insuficiente ética, agudización de la discrecionalidad laboral de los directivos para apoyar a sus amigos, contratos laborales temporales, rivalidad profesional, ausencia de reconocimientos e incentivos (Cladellas, Castelló y Parrado, 2018; El-Sahili, 2015; llaja y Reyes, 2016). Tercero, los factores extralaborales como: mercantilización del mundo, neofilia, neocultura, crisis matrimoniales, doble rol laboral y doméstica y conflictos trabajo y familia (El-Sahili, 2015; Gómez et al, 2019). Todo lo consignado son causales del SB del profesor universitario; por lo tanto, se justifica realizar investigaciones al respecto, para conocer el comportamiento del SB en muestras de diferentes países y realizar propuestas comunes para que se proporcione mejores condiciones laborales, que permitan un desempeño saludable y eficiente del profesor.

Es así que, para comprender la problemática existente con base en lo descrito anteriormente, es necesario tener claro la definición del SB, que es un problema de salud mental y ocupacional que se está incrementando y dificulta el desarrollo profesional del profesor universitario (Rodríguez-García, Sola-Martínez y Fernández-Cruz, 2017). Gil-Monte y Peiró (1999), definen el burnout estar quemado por el trabajo, como una respuesta al estrés laboral crónico, caracterizado por una vivencia de encontrarse emocional y físicamente agotado, actitudes y sentimientos negativos hacia las personas con las que se trabaja (despersonalización) y hacia el propio rol profesional (falta de realización personal en el trabajo). También existe un consenso entre diferentes autores (Gil-Monte y Moreno-Jiménez, 2007; Sánchez-Anguita, 2008; Tonon, 2003), respecto a considerar que el burnout como psicopatología laboral, comprende tres dimensiones básicas que son: 1) Agotamiento o cansancio emocional, es el síntoma más evidente, que cursa con la disminución y pérdida de recursos emocionales, cansancio y sensación de agotamiento.2) Despersonalización, se refiere a una respuesta impersonal de frialdad afectiva en el trato hacia los demás y actitudes negativas de insensibilidad y cinismo hacia los receptores del servicio prestado. 3) Bajo sentimiento de realización personal en el trabajo, es la disminución de sentimientos de competencia y éxito profesional en la tarea que se realiza cotidianamente; es decir es evaluar el propio trabajo de forma negativa con el autoreproche de no haber logrado los objetivos propuestos, acompañado de una percepción de baja autoestima profesional. 
De otra parte, en este estudio para explicar los resultados se asumieron dos modelos teóricos sobre el estrés laboral y uno sobre el burnout. El modelo demanda, control y apoyo social sostiene que las altas demandas y exigencias del trabajo y el bajo control para responder a dichas demandas, genera tensión emocional y repercute en la salud del trabajador (Karasek y Theorell, 1992). El modelo desbalance esfuerzo-recompensa de Siegrist (1996), establece que si no hay correspondencia entre el esfuerzo realizado en el trabajo y la recompensa obtenida (dinero, estima, status laboral), genera preocupación o ansiedad asociada a tensión. El modelo tridimensional del burnout de perspectiva psicosocial, señala que el SB comprende tres dimensiones que aparecen secuencialmente: cansancio emocional y físico, despersonalización (actitudinal) y baja realización personal en el trabajo (cognitivo) (Maslach, 1982).

Se indica que hay estudios internacionales precedentes sobre el SB en profesores universitarios, que han estudiado las características y niveles del SB y sus dimensiones; además otros estudios han confirmado la asociación de esta variable con otras, como la satisfacción laboral, intención de abandono, calidad de vida, desempeño laboral, estrés laboral, clima laboral, riesgos psicosociales, calidad educativa, sentimientos de autoeficacia, estrategias de inteligencia emocional, conflicto de roles docenteinvestigador (Arquero y Donoso, 2013; Brito, 2018; Bustamante, Bustamante, González y Bustamante, 2016; Cárdenas, Méndez y González, 2014; Carlotto y Gonçalves, 2017; Ferrel, Pedraza y Rubio, 2010; García, Escorcia y Pérez, 2017; Gómez, Perilla y Hermosa, 2015; Ho, 2017; Ibarra-Luna, Erazo-Muñoz y Gallego-López, 2018; llaja y Reyes, 2016; Jiménez, Arrieta y Quintero, 2017; López, 2014; Lou y Chen, 2016; Martins de Paiva, Do Nascimento y Helal, 2015; Martínez, Moreno y Ferrer, 2009; Mena, 2010; Moreno-Jiménez et al, 2009; Ocaña et al, 2015; Ponce, Bulnes, Aliaga, Atalaya y Huerta, 2005; Rivera et al, 2018; Rojas y Grisales, 2011; Xu, 2019; Zhang y Zhu, 2008); sin embargo no se ha realizado estudios comparativos sobre los niveles del burnout asociados a la carga docente e investigadora en profesores universitarios de países iberoamericanos, en este caso Perú y España, por lo que se considera que los resultados de este estudio, constituye un aporte importante para una mejor comprensión del SB e implementación de políticas y programas para su prevención, control y atenuación.

Con base en lo expuesto, se plantea como objetivo principal: analizar las diferencias del síndrome de burnout, sus dimensiones, tanto en la actividad de docencia como de investigación, entre los profesores de las Facultades de Ciencias Empresariales, según universidad de procedencia: Universidad de Sevilla y Universidad Nacional Santiago Antúnez de Mayolo; además identificar las diferencias de los resultados de las variables en mención, según variables sociodemográficas: sexo y edad de los evaluados.

\section{METODOLOGÍA}

El estudio es de enfoque cuantitativo, de nivel básico y descriptivo; además, es de diseño no experimental, transversal y descriptivo comparativo (Supo y Zacarías, 2020), pues de acuerdo a los objetivos propuestos, se estableció diferencias entre los niveles del síndrome de burnout, sus dimensiones, burnout por actividad de docencia y burnout por actividad de investigación, entre los profesores de las Facultades de Ciencias Empresariales, de la Universidad de Sevilla y de la Universidad Nacional Santiago Antúnez de Mayolo, según indicadores sociodemográficos. 
La población para el presente trabajo de investigación estuvo conformada por 89 docentes, de las Facultades de Ciencias Empresariales (Economía, Contabilidad, Administración y Turismo) 46 de la UNASAM (Perú) y 43 de la US (España). El tipo de muestreo fue no probabilístico, de tipo intencional; es decir de acuerdo a los conocimientos y criterios de los investigadores, respecto a la población de estudio, se formaron dos grupos considerando la disciplina profesional de profesores universitarios; por lo tanto, la muestra se conformó por un total de 68 sujetos (34 sujetos de cada universidad). Los criterios de inclusión fueron: profesores universitarios de ambos sexos, entre 25 a 70 años de edad, nombrados y contratados, que aceptaron participar voluntariamente. Los criterios de exclusión fueron: no considerar a los docentes que estuvieron de licencia durante el trabajo de campo y que no cumplieron con los criterios de inclusión.

El instrumento utilizado para recoger información, fue el cuestionario de medición del síndrome de quemarse en el trabajo (burnout) en profesores universitarios, el cual es una adaptación del MBI de Maslach y Jackson (Seisdedos, 1997) realizada por Arquero y Donoso (2006), cuya modificación ha pasado por varias etapas en las que se ha contrastado el diseño de los ítems, la viabilidad de incluir determinadas cuestiones, etc. En dicha adaptación, una vez comprobada la versión consistente del instrumento, se llevó a cabo la prueba piloto en una muestra conformada por el profesorado del Departamento de Contabilidad y Economía Financiera de la US. De los resultados del estudio piloto y los comentarios de los profesores que participaron en él, se infirió que el $\mathrm{MBI}$ no tenía en cuenta la relación diferente que existía entre las funciones sustantivas (docencia e investigación) del profesor universitario, centrándose en la docencia y no considerar el factor que posiblemente tenía mayor incidencia en el estrés, como la investigación; por lo tanto, realizaron un segundo nivel de adaptación del MBI (agregaron 12 ítems a la prueba original) y psicométrico (determinaron la validez y confiabilidad del instrumento), y así obtuvieron por separado medidas de la incidencia del SB, a través de dos áreas: docencia e investigación. La adaptación psicométrica del cuestionario, se realizó con una muestra de 35 sujetos de ambos sexos ( 17 hombres y 18 mujeres), un poco más de la mitad de ellos tenían el grado de doctor con una edad media de 34 años. Con base en dicho grupo de evaluación, se determinó la fiabilidad a través del alfa de Cronbach para cada una de las escalas. Respecto a la validez de constructo, se analizaron las correlaciones entre las dimensiones consideradas en la evaluación del burnout, a través del Rho de Spearman, el cual indicó la existencia de correlaciones fuertes y significativas ( $p$-value: 0.001) (Arquero y Donoso, 2006).

Asimismo, antes de aplicar el instrumento señalado a la muestra pervana, se verificó las propiedades psicométricas del mismo, que fueron adecuadas; así la validez de contenido fue calculada mediante Lawhe $=1.000$; la fiabilidad determinada mediante Alfa de Cronbach $=0.687$; y la validez de constructo se delimito mediante el análisis factorial (Kaiser-Meyer-Olkin de adecuación de muestreo $=0.703$, aprox. Chi cuadrado $=1823.155$ y prueba de esfericidad de Barlett $=630 \mathrm{Gl}$, sig 0.000 . 


\section{RESULTADOS}

Se obtuvo la información sociodemográfica de las muestras en función al sexo y la edad (tabla I). En la facultad de Ciencias Empresariales de la Universidad de Sevilla, la distribución de los casos fue homogénea respecto al sexo $158.82 \%$ varones y $41.18 \%$ mujeres), a excepción de la edad, dado que el $61.77 \%$ de los profesores tenía 35 o menos años. Así mismo, participo solamente un profesor en la categoría de 51 a 55 años, por lo que el puntaje en este rango es solo uno y no presenta variación. Mientras que, en la facultad de Ciencias Empresariales de la Universidad Santiago Antúnez de Mayolo, el $55.88 \%$ de los docentes tenía más de 55 años (entre 56 a 70 años) y no hubo profesores de menos de 35 años. Asimismo, las mujeres fueron representadas solamente por el $5.88 \%$ en la muestra de este estudio.

Tabla I. Distribución de la muestra según universidad, sexo y edad

\begin{tabular}{|c|c|c|c|c|c|}
\hline \multirow{2}{*}{\multicolumn{2}{|c|}{ Variables }} & \multirow{2}{*}{$\mathrm{n}$} & \multirow{2}{*}{$\%$} & \multicolumn{2}{|c|}{ Nivel de SB } \\
\hline & & & & Media & $\mathrm{DE}$ \\
\hline \multicolumn{6}{|c|}{ Universidad Sevilla } \\
\hline \multirow{2}{*}{ Sexo } & Varón & 20 & 58.82 & 93.50 & 13.06 \\
\hline & Mujer & 14 & 41.18 & 88.21 & 11.61 \\
\hline \multirow{7}{*}{ Edad } & Menos de 30 años & 8 & 23.53 & 84.63 & 9.24 \\
\hline & 30 a 35 años & 13 & 38.24 & 97.08 & 14.21 \\
\hline & 36 a 40 años & 5 & 14.71 & 95.80 & 7.60 \\
\hline & 41 a 45 años & 3 & 8.82 & 89.00 & 9.17 \\
\hline & 46 a 50 años & 2 & 5.88 & 84.50 & 23.33 \\
\hline & 51 a 55 años & $1^{*}$ & 2.94 & 79.00 & \\
\hline & 56 a 70 años & 2 & 5.88 & 86.00 & 7.07 \\
\hline \multicolumn{6}{|c|}{ Universidad Santiago Antúnez de Mayolo } \\
\hline \multirow{5}{*}{ Sexo } & Varón & 32 & 94.12 & 118.06 & 11.09 \\
\hline & Mujer & 2 & 5.88 & 101.50 & 3.54 \\
\hline & Menos de 30 años & 0 & & & \\
\hline & 30 a 35 años & 0 & & & \\
\hline & 36 a 40 años & 4 & 11.76 & 113.25 & 14.71 \\
\hline \multirow[t]{4}{*}{ Edad } & 41 a 45 años & 3 & 8.82 & 118.00 & 9.54 \\
\hline & 46 a 50 años & 5 & 14.71 & 110.80 & 7.66 \\
\hline & 51 a 55 años & 3 & 8.82 & 108.00 & 6.93 \\
\hline & 56 a 70 años & 19 & 55.88 & 120.84 & 11.55 \\
\hline
\end{tabular}

Nota: * Este valor es único y constante.

Previo al análisis de los datos, se realizó el análisis de la distribución de la muestra como se muestra en la tabla II; a un nivel de confianza del $99 \%$ se observa, que las dimensiones de cansancio emocional por docencia, cansancio emocional por investigación, despersonalización por investigación y la realización personal por investigación tienen valores no significativos. En el caso de despersonalización por docencia y realización personal por docencia, se obtuvieron valores significativos donde $p<0.01$, por lo que se concluye que la distribución de la muestra no es la normal. Con base a estos resultados el análisis de los objetivos se realizó a través de estadísticos no paramétricos. 


\begin{tabular}{llll}
\multicolumn{4}{c}{ Tabla II. Prueba de normalidad } \\
\hline & \multicolumn{3}{l}{ Kolmogorov-Smirnov } \\
\cline { 2 - 4 } & Estadístico & gl & Sig. \\
\hline CE Docencia & 0.08 & 68 & 0.20 \\
DP Docencia & 0.15 & 68 & 0.00 \\
RP Docencia & 0.16 & 68 & 0.00 \\
CE Investigación & 0.08 & 68 & 0.20 \\
DP Investigación & 0.10 & 68 & 0.06 \\
RP Investigación & 0.11 & 68 & 0.06 \\
\hline
\end{tabular}

El análisis del objetivo general, que pretende analizar las diferencias del SB según sus dimensiones, por la actividad de docencia y de investigación, entre los profesores de las Facultades de Ciencias Empresariales, según universidad de procedencia: US y UNASAM; se realizó mediante la prueba $U$ de Mann-Whitney (tabla III) a un nivel de confianza del 99\%, y se encontró que existen diferencias significativas en la despersonalización por docencia $(U=168, p=0.00)$, en la baja realización personal por docencia $(U=211.50, p=0.00)$, en la despersonalización por investigación $(U=214$, $p=0.00)$ y en la baja realización personal por investigación $(U=122.50, p=0.00)$. De manera similar a un nivel de confianza del $95 \%$, se encuentran diferencias significativas en el cansancio emocional por docencia $(U=385, p=0.02)$, en el caso de del cansancio emocional por investigación no se presentan diferencias significativas ( $U=572, p=0.94$ ).

Tabla III. Diferencias de resultados según la universidad

\begin{tabular}{|c|c|c|c|c|c|c|}
\hline Dimensión & Universidad & Media & $\mathrm{DE}$ & $\begin{array}{l}\text { U Mann- } \\
\text { Whitney }\end{array}$ & z & $p$ \\
\hline \multirow{2}{*}{ CE Docencia } & US & 18.03 & 8.23 & \multirow{2}{*}{385.00} & \multirow{2}{*}{-2.37} & \multirow{2}{*}{0.02} \\
\hline & UNASAM & 22.06 & 7.05 & & & \\
\hline \multirow{2}{*}{ DP Docencia } & US & 3.79 & 3.35 & \multirow{2}{*}{168.00} & \multirow{2}{*}{-5.05} & \multirow{2}{*}{0.00} \\
\hline & UNASAM & 9.59 & 4.06 & & & \\
\hline \multirow{2}{*}{ RP Docencia } & US & 34.44 & 6.02 & \multirow{2}{*}{211.50} & \multirow{2}{*}{-4.51} & \multirow{2}{*}{0.00} \\
\hline & UNASAM & 41.03 & 4.50 & & & \\
\hline \multirow{2}{*}{ CE Investigación } & US & 23.50 & 9.52 & \multirow{2}{*}{572.00} & \multirow{2}{*}{-0.07} & \multirow{2}{*}{0.94} \\
\hline & UNASAM & 23.21 & 7.02 & & & \\
\hline \multirow{2}{*}{ DP Investigación } & US & 4.44 & 3.99 & \multirow{2}{*}{214.00} & \multirow{2}{*}{-4.48} & \multirow{2}{*}{0.00} \\
\hline & UNASAM & 9.47 & 3.84 & & & \\
\hline \multirow{2}{*}{ RP Investigación } & US & 26.97 & 8.76 & \multirow{2}{*}{122.50} & \multirow{2}{*}{-5.59} & \multirow{2}{*}{0.00} \\
\hline & UNASAM & 39.68 & 5.02 & & & \\
\hline
\end{tabular}

Con respecto a los resultados según la función sustantiva sea por docencia o investigación, se analizó la diferencia a través de la Z de Wilcoxon, y se encontró que

\footnotetext{
I Se considera baja realización personal, pues los ítems han sido invertidos
} 
en la UNASAM se evidencia a un nivel de confianza del 99\%, que existe diferencia significativa en el cansancio emocional $(z=2.96, p=0.00)$, y la mayor puntuación se presenta para la investigación; es decir los docentes de la UNASAM manifiestan tener cansancio emocional mayormente por realizar actividades académicas de investigación, que por la docencia. Del mismo modo se encuentran diferencias significativas en la realización personal $(\mathrm{Z}=4.25, \mathrm{p}=0.00)$; es decir la función de docencia tiene mayor puntuación, que la función de investigación; cabe mencionar que la diferencia es significativa, pero la diferencia entre los valores es pequeña (0.5). En la dimensión de despersonalización, no se evidencian diferencias significativas. En lo que respecta a la US se encuentra que a un nivel de confianza del $95 \%$, existe diferencias significativas en el cansancio emocional $(Z=2.22, p=0.03)$, que es similar a los resultados encontrados para la UNASAM, es decir la mayor puntuación es por realizar la función de investigación. En la dimensión de realización personal, también se encuentran diferencias significativas $(\mathrm{Z}=2.53, \mathrm{p}=0.01)$, para función de docencia es mayor la puntuación, que para la investigación. En lo que respecta a la despersonalización, no se evidencia diferencias significativas. Es preciso señalar que la diferencia de los resultados antes mencionados, respecto al cansancio emocional y la baja realización personal, es en mayor proporción para la US, en comparación a la UNASAM.

Tabla IV. Diferencias de resultados según la función de docencia e investigación por universidad

\begin{tabular}{llllcc}
\hline & Media & DE & dif (D-1) & $\begin{array}{c}\text { Z } \\
\text { Wilcoxon }\end{array}$ & P \\
\hline CE Docencia & 21.50 & 7.05 & -1.50 & 2.96 & 0.00 \\
CE Investigación & 23.00 & 7.02 & & & \\
D & 9.50 & 4.06 & 0.50 & 1.83 & 0.07 \\
DP Investigación & 9.00 & 3.84 & & & \\
RP Docencia & 41.00 & 4.50 & 0.50 & 4.25 & 0.00 \\
RP Investigación & 40.50 & 5.02 & & & \\
\hline CE Docencia & 16.50 & 8.23 & -4.50 & 2.22 & 0.03 \\
CE Investigación & 21.00 & 9.52 & & & \\
DP Docencia & 4.00 & 3.35 & 0.50 & 0.32 & 0.75 \\
DP Investigación & 3.50 & 3.99 & & & \\
RP Docencia & 35.50 & 6.02 & 8.50 & 2.53 & 0.01 \\
RP Investigación & 27.00 & 8.76 & & & \\
\hline
\end{tabular}

En cuanto a los resultados según el sexo de los evaluados, se analizó a través de la $U$ de Mann-Whitney a un nivel de confianza del $95 \%$, y como se muestra en la tabla $\mathrm{V}$, los resultados muestran que existen diferencias significativas en la baja realización personal por investigación $(\mathrm{U}=385, \mathrm{p}=0.02)$ en los resultados de la Universidad de Sevilla, donde los varones tienen mayor puntuación que las mujeres. En el resto de dimensiones evaluadas de los profesores de la US, no existe diferencias significativas. En 
el caso de los resultados de los profesores de la UNASAM no existe diferencias significativas en ningún caso.

Tabla V. Diferencias de resultados según sexo

\begin{tabular}{|c|c|c|c|c|c|c|}
\hline & Género & & Media & $\mathrm{DE}$ & $\begin{array}{l}\text { U de Mann- } \\
\text { Whitney }\end{array}$ & $p$ \\
\hline \multirow{12}{*}{ 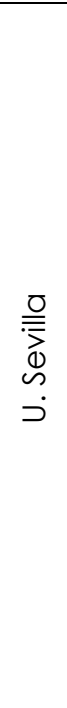 } & \multirow{2}{*}{ CE Docencia } & Varón & 18.25 & 8.29 & \multirow{2}{*}{124.50} & \multirow{2}{*}{0.59} \\
\hline & & Mujer & 17.71 & 8.45 & & \\
\hline & \multirow{2}{*}{ DP Docencia } & Varón & 3.55 & 2.78 & \multirow{2}{*}{137.00} & \multirow{2}{*}{0.92} \\
\hline & & Mujer & 4.14 & 4.11 & & \\
\hline & \multirow{2}{*}{ RP Docencia } & Varón & 35.95 & 5.29 & \multirow{2}{*}{90.00} & \multirow[b]{2}{*}{0.08} \\
\hline & & Mujer & 32.29 & 6.52 & & \\
\hline & \multirow{2}{*}{ CE Investigación } & Varón & 22.40 & 9.64 & \multirow{2}{*}{116.00} & \multirow{2}{*}{0.40} \\
\hline & & Mujer & 25.07 & 9.47 & & \\
\hline & \multirow{2}{*}{ DP Investigación } & Varón & 3.80 & 3.30 & \multirow{2}{*}{118.00} & \multirow{2}{*}{0.44} \\
\hline & & Mujer & 5.36 & 4.80 & & \\
\hline & \multirow{2}{*}{ RP Investigación } & Varón & 29.90 & 8.38 & \multirow{2}{*}{69.50} & \multirow{2}{*}{0.01} \\
\hline & & Mujer & 22.79 & 7.75 & & \\
\hline \multirow{12}{*}{ 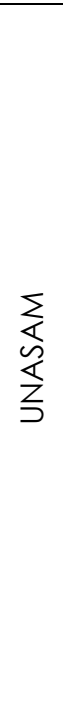 } & \multirow{2}{*}{ CE Docencia } & Varón & 22.31 & 7.17 & \multirow{2}{*}{18.50} & \multirow{2}{*}{0.32} \\
\hline & & Mujer & 18.00 & 2.83 & & \\
\hline & \multirow{2}{*}{ DP Docencia } & Varón & 9.66 & 4.08 & \multirow{2}{*}{26.00} & \multirow{2}{*}{0.66} \\
\hline & & Mujer & 8.50 & 4.95 & & \\
\hline & \multirow{2}{*}{ RP Docencia } & Varón & 41.22 & 4.32 & \multirow{2}{*}{24.00} & \multirow{2}{*}{0.56} \\
\hline & & Mujer & 38.00 & 8.49 & & \\
\hline & \multirow{2}{*}{ CE Investigación } & Varón & 23.56 & 7.08 & \multirow{2}{*}{14.50} & \multirow{2}{*}{0.20} \\
\hline & & Mujer & 17.50 & 2.12 & & \\
\hline & \multirow{2}{*}{ DP Investigación } & Varón & 9.47 & 3.79 & & \\
\hline & & Mujer & 9.50 & 6.36 & 31.00 & 0.94 \\
\hline & & Varón & 39.78 & 4.93 & 0050 & ת \\
\hline & RPInvestigacion & Mujer & 38.00 & 8.49 & 28.50 & 0.80 \\
\hline
\end{tabular}

En relación a los resultados según la edad de los evaluados (tabla VI), se encontró que la UNASAM no cuenta con profesores menores a 35 años, los dos primeros rangos de edad tienen una muestra igual a cero, es decir que no hay profesores en estas edades. Por tanto, el análisis de comparación de medias no pudo ser ejecutado para los grupos de menos de 30 años y el grupo de 30 a 35 años. Por otra parte, el rango de 51 a 55 años en la US no presenta variación, ya que el grupo presenta un valor constante de 1. Por este motivo, tampoco se realizó el análisis para grupos en este rango de edad; por ende, el análisis según la edad fue de solo 4 de los 7 rangos establecidos (36 a 40 años, 41 a 45 años, 46 a 50 años y de 56 a 70 años). Para el análisis de los resultados según la edad se utilizó la prueba $\mathrm{H}$ de Kruskal-Wallis, a un nivel de confianza del $95 \%$ no se evidencian diferencias significativas en ninguno de los casos, ni para la US, tampoco para la UNASAM. 
Tabla VI. Diferencias de resultados según la edad.

\begin{tabular}{|c|c|c|c|}
\hline & Dimensiones & $\begin{array}{c}\mathrm{H} \text { de Kruskal- } \\
\text { Wallis }\end{array}$ & $\mathrm{p}$ \\
\hline \multirow{6}{*}{ 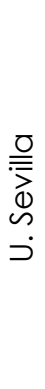 } & CE Docencia & 6.05 & 0.11 \\
\hline & DP Docencia & 5.32 & 0.15 \\
\hline & RP Docencia & 1.33 & 0.72 \\
\hline & CE Investigación & 2.85 & 0.42 \\
\hline & DP Investigación & 4.76 & 0.19 \\
\hline & RP Investigación & 1.32 & 0.72 \\
\hline \multirow{6}{*}{ 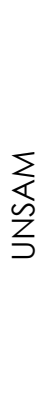 } & CE Docencia & 7.22 & 0.07 \\
\hline & DP Docencia & 0.47 & 0.93 \\
\hline & RP Docencia & 1.36 & 0.72 \\
\hline & CE Investigación & 5.98 & 0.11 \\
\hline & DP Investigación & 0.98 & 0.81 \\
\hline & RP Investigación & 0.82 & 0.85 \\
\hline
\end{tabular}

\section{DISCUSIÓN}

De los resultados encontrados, se infiere que los profesores evaluados de la Facultad de Ciencias Empresariales de la UNASAM presentan niveles significativamente más altos de SB en las dimensiones de cansancio emocional por docencia, despersonalización por docencia e investigación y en baja realización personal por docencia e investigación, en comparación a lo presentado por los profesores evaluados de la US. Este resultado es concordante con resultados de otros estudios realizados en muestras de profesores universitarios latinoamericanos (Brito, 2018; Bustamante et al, 2016; llaja y Reyes, 2016; Rojas y Grisales, 2011). Del mismo modo se evidencia la diferencia en lo que respecta al cansancio emocional y baja realización personal, por las funciones de docencia e investigación, siendo esta diferencia más remarcada en la US, en comparación a la UNASAM. Este resultado es concordante con lo hallado por Arquero y Donoso (2013), a su vez los resultados encontrados en este estudio preocupan, pues los niveles altos de burnout en este grupo ocupacional estaría asociado con el decremento del desempeño docente, la productividad laboral y la calidad educativa (Cárdenas et al, 2014; Jiménez et al, 2017; Martínez et al; 2009; Ocaña, 2017) y con la intención de abandono de la profesión (Arquero y Donoso, 2013; Moreno-Jiménez et al, 2009), este último afectaría a las universidades, ya que los docentes constituyen el capital humano formado para el ejercicio de la docencia universitaria y su deserción implica recursos potenciales perdidos. 
Para comprender los resultados hallados, es necesario tener en cuenta que el contexto socioeconómico, político, cultural y educativo es distinto de país a país; sin embargo, hay aspectos comunes para los países iberoamericanos como España y Perú, como el escaso presupuesto asignado a las universidades públicas, y las políticas públicas implementadas para las universidades en general (Aramburuzabala, Martínez-Garrido y García-Peinado, 2013; Bruner y Villalobos, 2014; Rodríguez, 2018; Arquero, Jiménez-Cardoso y Laffarga-Briones, 2018).

Asimismo, los resultados encontrados revelan que los profesores evaluados de la US y de la UNASAM, por igual presentan cansancio emocional por investigación sin diferencias significativas; se infiere que la dedicación a la investigación desgasta indistintamente a los docentes de la UNASAM y de US, aunado a la falta de recompensas contingentes suficientes por el rendimiento en investigación. También los niveles de despersonalización y baja realización personal, tanto por realizar la función de docencia, como por investigación de los profesores de la UNASAM, son significativamente más altos, que de los profesores de la US; este resultado se explicaría en parte, porque los profesores de UNASAN con frecuencia realizan simultáneamente docencia e investigación, están en mayor contacto con los estudiantes, hacia quienes muestran sentimientos y actitudes negativas, trato impersonal, poco interés y empatía, que de una parte afecta la evaluación de desempeño de los profesores (Jiménez et al, 2017), con menor reconocimiento de la labor docente de parte del estudiantado; $y$ de otra la percepción de la calidad de los servicios educativos.

También, el profesor de la UNASAM presenta mayor tendencia a evaluarse negativamente, puede sentirse descontento e incompetente consigo mismo al ejercer la docencia universitaria, que se relacionaría con la disminución del desempeño docente (Cárdenas et al, 2014) y con la intención de abandono de la profesión docente o de la universidad (Arquero y Donoso, 2013; Martínez et al, 2009). Diferentes estudios realizados a nivel internacional sobre burnout en profesores universitarios, confirman que este grupo ocupacional además de presentar cansancio emocional, evidencia niveles altos de despersonalización y baja realización personal (Brito, 2018; Ibarra-Luna et al, 2018; Jiménez et al, 2017; Rojas y Grisales, 2011; Correa, Muñoz y Chaparro, 2010).

Con respecto a los resultados según el sexo, se evidencian diferencias en la baja realización personal por investigación en los profesores de la US, teniendo mayor puntuación los varones a diferencia de las mujeres; dicha diferencia es mínima pero significativa lo que nos indica que los profesores de la US frente a la actividad de investigar son afectados de manera negativa sobre su realización personal, perjudicando en mayor grado a los varones que a las mujeres, en el resto de dimensiones tanto por docencia como investigación, no existen diferencias significativas. Este hallazgo coincide con resultados hallados en estudios precedentes internacionales (Chavarría, Colunga, Loria y Peláez, 2017; Ibarra-Luna et al, 2018; Lou y Chen, 2016; Mena, 2010; Ponce et al, 2005); pero otros estudios hallaron lo contrario, mayor SB sea en las mujeres o en varones (Arquero y Donoso, 2013; Correa et al, 2010; Ocaña et al, 2015). Además cabe indicar que casi la mitad de la muestra para la US son mujeres y para la UNASAM solamente el 5.88\%; lo cual indicaría que en la US adaptada a un contexto cultural europeo menos tradicional, hay mayor equidad de género y políticas institucionales que no afectarían el desarrollo de la carrera 
académica de las profesoras; en cambio en el Perú persiste una cultura conservadora, y mayormente la elección y ejercicio de las carreras empresariales estarían relacionadas con el predominio de la ideología machista; y también en la UNASAM no se han implementado políticas institucionales y programas de promoción de equidad de género y de apoyo para el desarrollo de la carrera académica (docencia e investigación).

En referencia a los resultados según la edad, se evidencia que no existe diferencias significativas en los resultados de cada una de las dimensiones (docencia e investigación), es decir que el paso de los años y el tiempo de servicio laboral, no afectaría la capacidad de afrontamiento y manejo de SB, y asimismo presentarían recursos de adaptación similares (Mateo, 2013), lo cual se relaciona con el desempeño docente (Cárdenas et al, 2014; García, Escorcia y Pérez, 2017). Sin embargo, los resultados no serían concluyentes para la variable edad, pues estudios precedentes registraron asociación y no asociación entre la variable edad y el SB (Chavarría et al, 2017; Gil-Monte, 2006). Cabe indicar que la mayoría (88.23\%) de la muestra de la UNASAM son adultos intermedios y mayores de 41 a 70 años de edad, y contrariamente la mayoría $(76.48 \%)$ de la muestra de la US, son profesores adultos tempranos.

Para el caso de profesores adultos tempranos e intermedios, la presencia de SB ya sea por docencia o investigación, se debería a que este grupo mayormente enfrenta estresores como contratos temporales, bajos salarios, inestabilidad laboral, excesiva carga de trabajo sobre todo en la docencia, evaluación periódica para ratificación de contrato, asenso y promoción docente, y el deseo de calificar como docente investigador, que permite acceder al bono económico especial. Estos resultados difieren de lo encontrado por Arquero y Donoso (2013) e llaja y Reyes (2016), quienes reportaron que el nivel alto de SB en los profesores universitarios de España y Ecuador, está asociada a las actividades de investigación, que a la docencia. Asimismo, el conflicto de roles docente-investigador estaría asociado con el SB y sus dimensiones de cansancio emocional y despersonalización (XU, 2019).

Con relación a los profesores adultos mayores, la mayoría tienen estabilidad laboral, ostentan categorías docente asociado o principal con mayor remuneración; sin embargo enfrentan crónicamente estresores laborales, y están expuestos a otros estresores propios de la edad, como estar próximos a la jubilación; tener mayores problemas de salud que merma la vitalidad; dificultad para adaptarse al uso de las nuevas tecnologías de información y comunicación y al entorno virtual, en el proceso enseñanza-aprendizaje e investigación; cumplir en plazos establecidos otras exigencias burocráticas para fines de evaluación, ratificación docente, licenciamiento y acreditación institucional, que restan tiempo para cumplir con eficiencia las actividades académicas, demandas sociofamiliares, como para el descanso, recreación, lo cual genera mayor SB, que impacta en la productividad, salud ocupacional, bienestar y calidad de vida del profesor universitario.

Los resultados presentados en este estudio tienen limitaciones metodológicas, pues es un estudio ex post facto, cuyo muestreo es de tipo no probabilístico, y la muestra reducida; por lo tanto, los resultados son válidos para la muestra de estudio, no son 
generalizables. Se sugiere realizar estudios de revisión de las propiedades psicométricas del instrumento de investigación con muestras más amplias y reducir el número de ítems, para medir específicamente el SB por docencia e investigación. Además es pertinente llevar a cabo investigaciones transculturales con muestras de otros países iberoamericanos, para confirmar las tendencias encontradas respecto al SB por actividades de docencia e investigación, y estudios correlacionales sobre las variables sociodemográficas y moderadoras asociadas al SB por docencia e investigación, para propuestas de políticas de protección de salud ocupacional e implementación de programas de prevención e intervención para controlar y reducir los niveles del SB, que afecta el desarrollo profesional y bienestar de los profesores universitarios.

\section{REFERENCIAS}

Aramburuzabala, P., Martínez-Garrido, C. y R. García-Peinado (2013): "La formación del profesorado universitario en España: evolución y perspectivas". Educación, 22(43), pp. 7-25.

Arquero, J. y J. Donoso (2006): "Instrumento de medición del "síndrome de quemarse en el trabajo" (Burnout) en profesores universitarios", Publicación Contabilidad y Auditoría, 23, pp.1-14. Disponible en: https://idus.us.es/bitstream/handle/11441/78806/ Instrumento\%20de\%20medici\%c3\%b3n\%20del\%20sindrome\%20de\%20quemarse \%20en\%20el\%20trabajo.pdf? sequence=1 \&isAllowed=y [Consulta: 28 de abril de 2020].

Arquero, J. y J. Donoso (2013): "Docencia, investigación y burnout: el síndrome del quemado en profesores universitarios de contabilidad", Revista de contabilidad. 6(2), pp. 94-105. Disponible en: https://doi.org/10.1016/j.rcsar.2013.04.001 [Consulta: 28 de abril de 2020].

Arquero, J., Jiménez-Cardoso, S. y J. Laffarga-Briones (2018): "Patrones generales de investigación en contabilidad: análisis de una muestra de universidades". EDUCADE, 9, pp. 43-64.

Avargues, M. y M. Borda (2010): "Estrés laboral y síndrome de burnout en la Universidad: Análisis descriptivo de la situación actual y revisión de las principales líneas de investigación", Anuario de Psicología Clínica y de la Salud, 6, pp. 73-78.

Botero, C. (2012): "Riesgo psicosocial intralaboral y "burnout" en docentes universitarios", Cuadernos de Administración, 28 (48), pp. 117-132.

Bruner, J. y C. Villalobos (2014): "III Encuentro de Rectores Universia, Río de Janeiro, 2014. Políticas de educación superior en Iberoamérica, 2009-2013". Ediciones Universidad Diego Portales, Santiago de Chile. Disponible en: https://virtualeduca.org/documentos/centrodocumentacion/2014/IIIencuentro-politicas-ibe-2009-2013.pdf [Consulta: 28 de abril de 2020].

Brito, J. (2018): "Calidad educativa en las instituciones de educación superior: evaluación del síndrome de burnout en los profesores", Revista Iberoamericana para la Investigación y el Desarrollo, 8(16), pp. 516-534. Disponible en: https://doi.org/10.23913/ride.v8i16.356 [Consulta: 28 de abril de 2020]. 
Bustamante, E., Bustamante F., González, G. y L. Bustamante (2016): "El burnout en la profesión docente: un estudio en la escuela de bioanálisis de la Universidad de Carabobo Sede Aragua, Venezuela", Medicina y Seguridad del Trabajo, 62(243), p. 111-121.

Cárdenas, M., Méndez, L. y M. González (2014): "Evaluación del desempeño docente, estrés y burnout en profesores universitarios", Actualidades Investigativas en Educación, 14(1), pp. 1-22. Disponible en: https://doi.org/10.15517/aie.v14i1.13210 [Consulta: 28 de abril de 2020].

Carlotto, M y S. Gonçalves (2017): "Riscos psicossociais associados à síndrome de burnout em professores universitários", Avances en Psicología Latinoamericana, 35(3), pp. 447-457. Disponible en: http://dx.doi.org/10.12804/10.12804/ [Consulta: 28 de abril de 2020].

Chavarría, R., Colunga, F., Loria, J. y K. Peláez (2017): "Síndrome de burnout en médicos docentes de un hospital de $2^{\circ}$ nivel en México", Educación Médica, 18(4), pp. 254-261.

Cladellas, R., Castelló, A. y E. Parrado (2018): "Satisfacción, salud y estrés laboral del profesorado universitario según su situación contractual", Revista Salud Pública, 20(1), pp. 53-59.

Conceição, R., Pastore da Silveira, A., Barbosa de Sá, M., De Barros, S., Silva, J. y A. De Barros (2015): "Transtorno mental e estressores no trabalho entre profesores universitários da área da saúde", Trabalho, Educação e Saúde, 13(1), pp. 135155.

Correa, Z., Muñoz, I. y A. Chaparro (2010): "Síndrome de burnout en docentes de dos universidades de Popayán, Colombia", Revista de salud pública, 12(4), pp. 589598. Disponible en: https://doi.org/10.1590/s0124-00642010000400006 [Consulta: 28 de abril de 2020].

Díaz, A. (2014): Influencia del Síndrome de Burnout en el desempeño docente de los profesores de la Facultad de Ciencias de la Salud de la Universidad Nacional del Callao-2013, Tesis Doctoral, Universidad Nacional Mayor de San Marcos, Perú.

El-Sahili, L. (2015): Burnout consecuencias y soluciones, México D.F., Manual Moderno.

Fajardo, A., Montejo, F., Molano, G., Hernández, J. y A. Quintero (2013): "Correlación entre los factores de riesgo intralaboral y los niveles séricos de cortisol en docentes universitarios", Ciencia \& Trabajo, 15(46), pp. 1-6.

Ferrel, R., Pedraza, C. y B. Rubio (2010): "El síndrome de quemarse en el trabajo (burnout) en docentes universitarios", DUAZARY, 7 (1), pp. 15-28.

García, A., Escorcia, C. y B. Pérez (2017): "Burnout syndrome and self-efficacy beliefs in professors", Propósitos y Representaciones, 5(2), pp. 65 - 126. Disponible en: https://doi.org/10.2051 1/pyr2017.v5n2.170 [Consulta: 28 de abril de 2020].

García, M., Iglesias, S., Saleta, M. y J. Romay (2016): "Riesgos psicosociales en el profesorado de enseñanza universitaria: diagnóstico y prevención", Journal of 
Work and Organizational Psychology, 32(3), pp. 173-182. Disponible en: https://doi.org/10.1016/.r.rpto.2016.07.001 [Consulta: 28 de abril de 2020].

Gil-Monte, P. (2006): El síndrome de quemarse por el trabajo (Burnout). Una enfermedad laboral en la sociedad del bienestar, Madrid, Ediciones Pirámide.

Gil-Monte, P. y J. Peiró (1999): "Perspectivas teóricas y modelos interpretativos para el estudio del síndrome de quemarse por el trabajo", Anales de psicología, 15 (2), pp. 261-268.

Gil-Monte, P. y B. Moreno-Jiménez (2007): El síndrome de quemarse por el trabajo (Burnout). Grupos profesionales de riesgo, Madrid, Ediciones Pirámide.

Gómez, V., Perilla, L. y A. Hermosa (2015): "Moderación de la relación entre tensión laboral y malestar de profesores universitarios: papel del conflicto y la facilitación entre el trabajo y la familia", Revista Colombiana de Psicología, 24(1), pp. 185-201. Disponible en: https://doi:10.15446/rcp.v24n1.42081 [Consulta: 28 de abril de 2020].

Gómez, V., Perilla, L. y A. Hermosa (2019): "Riesgos para la salud de profesores universitarios derivados de factores psicosociales laborales", Universitas Psychologica, 18(3), pp. 1-5. Disponible en: https://doi.org/10.11144/Javeriana.upsy 18-3.rspu [Consulta: 28 de abril de 2020].

Ho, S. (2017): "The relationship between teacher stress and burnout in Hong Kong: positive humour and gender as moderators", Educational Psychology, 37(3), pp. 272-286. Disponible en: $\quad$ https://doi.org/10.1080/01443410.2015.1120859 [Consulta: 28 de abril de 2020].

Ibarra-Luna, M., Erazo-Muñoz, P. y F. Gallego-López (2018): "Síndrome de burnout en profesores de una institución de educación superior de Manizales-Colombia", Revista de Investigaciones UCM, 18(32), pp. 69-83.

llaja, B. y C. Reyes (2016): "Burnout y estrategias de inteligencia emocional en profesores universitarios: implicaciones en la salud laboral educativa", Psicología desde el Caribe, 33(1), pp. 31-46. Disponible en: https://doi.org/10.14482/psdc.33.1.8081 [Consulta: 28 de abril de 2020].

Jiménez, L., Arrieta, M. y K. Quintero (2017): "Síndrome de Burnout y evaluación de desempeño en docentes de la Fundación Universitaria del área andina sede Valledupar", Escenarios, 15(1), pp. 36-46. Disponible en: http://dx.doi.org/10.15665/esc.v15i1.1120 [Consulta: 28 de abril de 2020].

Karasek, R. y T. Theorell (1992): Healthy work: stress, productivity and de reconstruction of working life, New York, Basic Books.

López, B. (2014): Clima laboral y el síndrome de burnout de los docentes de la Facultad de Ciencias Sociales, Educación y de la Comunicación, 2014, Tesis de Maestría, Universidad Nacional Santiago Antúnez de Mayolo, Perú.

Lou, Y. Y L. Chen (2016): "A Study of the English Teachers' burnout in a Local Comprehensive University in China", Creative Education, 7, pp. 646-654. Disponible en: http://dx.doi.org/10.4236/ce.2016.74067 [Consulta: 28 de abril de 2020]. 
Martínez, M., Moreno., B. y R. Ferrer (2009): Calidad de vida del profesorado universitario: el desgaste profesional. (Propuesta metodológica y resultados). Acciones e Investigaciones Sociales, (27), pp. 131-169. Disponible en:https://doi.org/10.26754/ojs_ais/ais.200927346 [Consulta: 28 de abril de 2020].

Martins de Paiva, K., Do Nascimento, M. y D. Helal (2015): "Estresse ocupacional e síndrome de burnout: proposição de um modelo integrativo e perspectivas de pesquisa junto a docentes do ensino superior", Gestão \& Planejamento, 16(3), pp. 285-309.

Maslach, C. (1982): Burnout: the cost of caring, Englewood Cliffs, New Jersey, Prentice Hall.

Mateo, P. (2013): Control de estrés laboral, España, FC Editorial.

Mena, L. (2010): El desgaste profesional en profesores universitarios: un modelo predictivo, España, Editorial de la Universidad de Granada.

Monarca, H. y M. Prieto (2018): Calidad de la educación superior en Iberoamérica, Madrid, Editorial Dykinson.

Moreno-Jiménez, B., Garrosa, E. y R. Rodríguez (2009): "El burnout del profesorado universitario y las intenciones de abandono: un estudio multi-muestra", Revista de Psicología del Trabajo y de las organizaciones, 25(2), pp. 149-163.

Ocaña, M. (2017): El burnout y su incidencia en la productividad docente, Tesis de Licenciatura, Universidad Estatal de Milagro, Ecuador.

Ocaña, R., Rodríguez, A., Urbina, M. y O. Sánchez (2015): "Prevalencia del síndrome de burnout en el personal docente que labora en el centro universitario UAEM Zumpango", Congreso Internacional de Investigación Academia Journals Celaya, Guanajuato, México.

Ponce, C., Bulnes, M., Aliaga, J., Atalaya, M. y R. Huerta (2005): "El síndrome del "Quemado" por estrés laboral asistencial en grupos de docentes universitarios", Revista de investigación en psicología, 8(2), pp. 85-112.

Rivera, A., Segarra, P. y G. Giler (2018): "Síndrome de burnout en docentes de instituciones de educación superior", AVFT Archivos venezolanos de farmacología y terapéutica, 37(2), pp. 78-84.

Rodríguez, L. (2018): "Docencia de contabilidad internacional en el sistema universitario español. Metodologías docentes y sistemas de evaluación", EDUCADE, 9, pp. 5-32.

Rodríguez-García, A., Sola-Martínez, T. y M. Fernández-Cruz (2017): "Impacto del burnout en el desarrollo profesional del profesorado universitario. Una Revisión de la investigación", Revista Electrónica Interuniversitaria de Formación del Profesorado, 20(3), pp. 161-178. Disponible en: http://dx.doi.org/10.6018/reifop.20.3.275121 [Consulta: 28 de abril de 2020].

Rojas, M. y H. Grisales (2011): "Burnout syndrome in professors from an academic unit of a Colombian university", Investigación y Educación en Enfermería, 29(3), pp. 427-434. 
Sánchez-Anguita, A. (2008): Psicopatologías laborales, Salamanca: Publicaciones Universidad Pontificia Salamanca.

Seisdedos, N. (1997): Introducción, normalización y comentarios a la versión española del MBI. Maslach, C. y Jackson, S.E, Madrid, TEA.

Siegrist, J. (1996): "Adverse health effects of high-effort/low-reward conditions", Journal of Occupational Health Psychology, 1(1), pp. 27-41.

Supo, J. y H.Zacarias (2020). Metodología de la investigación científica: Para las ciencias de la salud y las ciencias sociales, Perú, Sincie.

Tonon, G. (2003): Calidad de vida y desgaste profesional. Una mirada del síndrome de Burnout, Buenos Aires, Espacio Editorial.

Villamar, D., Juárez, A., Gonzáles, I. y M. Osnaya (2019): "Psychosocial factors and burnout syndrome in academics of a public university from México", Propósitos y Representaciones, 7(3), pp. 111-140.

XU, L. (2019): "Teacher-researcher role conflict and burnout among Chinese university teachers: a job demand-resources model perspective", Studies in Higher Education, 44(6), pp. 903-919. Disponible en: https://doi.org/10.1080/03075079.2017.1399261 [Consulta: 28 de abril de 2020].

Zhang, Q. y W. Zhu (2008): "Exploring emotion in teaching: Emotional labor, burnout, and satisfaction in Chinese higher", Communication Education, 57(1), pp. 105122. Disponible en: https://doi.org/10.1080/03634520701586310 [Consulta: 28 de abril de 2020]. 